\title{
Co-existence of endometriosis with 13 non-gynecological co-morbidities: Mutation analysis by whole exome sequencing
}

\author{
CHAROULA MATALLIOTAKI ${ }^{1,2}$, MICHAIL MATALLIOTAKIS ${ }^{1,2}$, MARIA I. ZERVOU ${ }^{3}$, \\ ALEXANDRA TRIVLI $^{3,4}$, IOANNIS MATALLIOTAKIS ${ }^{2}$, GEORGE MAVROMATIDIS $^{1}$, \\ DEMETRIOS A. SPANDIDOS ${ }^{5}$, HANS M. ALBERTSEN ${ }^{6}$, RAKESH CHETTIER ${ }^{6}$, \\ KENNETH WARD ${ }^{6}$ and GEORGE N. GOULIELMOS ${ }^{3}$
}

\author{
${ }^{1}$ 3rd Department of Obstetrics and Gynecology, Aristotle University of Thessaloniki, Thessaloniki 54124; \\ ${ }^{2}$ Department of Obstetrics and Gynecology, Venizeleio General Hospital of Heraklion, Heraklion 71409; \\ ${ }^{3}$ Section of Molecular Pathology and Human Genetics, Department of Internal Medicine, School of Medicine, \\ University of Crete, Heraklion 71003; ${ }^{4}$ Department of Ophthalmology, Konstantopouleio-Patission \\ General Hospital, Athens $14233 ;{ }^{5}$ Laboratory of Clinical Virology, Medical School, University of \\ Crete, Heraklion 71003, Greece; ${ }^{6}$ Juneau Biosciences, Salt Lake City, UT 84109, USA
}

Received July 23, 2018; Accepted September 14, 2018

DOI: $10.3892 / \mathrm{mmr} .2018 .9521$

\begin{abstract}
Endometriosis is an enigmatic condition with an unknown etiology and poorly understood pathogenesis and women with endometriosis represent a high-risk population group for a large category of chronic conditions. The study focused on a 67-year-old woman who presented with a 40-year history of familial endometriosis associated with various non-gynecological co-morbidities, thus representing a unique case from a cohort of 1,000 patients with endometriosis. Her family history included infertile members suffering from endometriosis. Thirteen non-gynecological co-morbidities were documented throughout the years, including five autoimmune diseases (i.e., systemic lupus erythematosus, ankylosing spondylitis, multiple sclerosis, bronchial asthma and Crohn's disease), urinary bladder diverticulum, osteoporosis, multinodular goiter, cardiovascular diseases, gastroesophageal reflux disease, malignant tumor of urinary bladder, Barrett's esophagus and bilateral cataract. In order to understand the potential role of gene mutations in the development of all those co-morbidities, whole exome sequencing was performed and the presence of various disease-associated, potentially causal missense variants, were observed. These findings are in accordance with the previously suggested common underlying etiologic pathway for
\end{abstract}

Correspondence to: Dr George N. Goulielmos, Section of Molecular Pathology and Human Genetics, Department of Internal Medicine, School of Medicine, University of Crete, Voutes, Heraklion 710 03, Crete, Greece

E-mail: goulielmos@med.uoc.gr

Key words: co-morbidities, endometriosis, family history, autoimmune diseases, whole exome sequencing some, but not all, autoimmune disorders. This unusual case provides novel insights demonstrating that endometriosis can coexist with various chronic autoimmune diseases and other conditions, including non-gynecological malignancies, which possibly share a common genetic cause, a fact that should be taken into consideration seriously by clinicians.

\section{Introduction}

Endometriosis is an enigmatic condition with an unknown etiology and poorly understood pathogenesis. It is defined by the presence of endometrial tissue external to the uterine cavity and women with endometriosis represent a high-risk population group for a large category of chronic conditions (1). Familial association of endometriosis additionally suggests a genetic contribution to the disease and various genetic susceptibility loci have been identified by case-control studies as well as genome-wide association studies (GWAS) (2).

Findings related to the co-morbidities of gynecological and non-gynecological diseases with endometriosis have been published thus far (3). In this framework, diverse associations between endometriosis and other diseases were examined including autoimmune and endocrine disorders, cardiovascular diseases, benign gynecological conditions and various types of malignancy (3). The latter is expected considering that endometriosis shares some characteristics with malignant tumors, such as invasion of local and distant organs, abnormal tissue growth, dysfunction of target organs, and genetic damage. Moreover, previous case reports studies emphasized on co-morbidities of endometriosis with various autoimmune disorders, including autoimmune alopecia universalis, autoimmune thyroiditis, multiple sclerosis (MS), and autoimmune progesterone dermatitis (4). In addition, similarities between systemic lupus erythematosus (SLE) and endometriosis were detected upon a comparative evaluation of clinical and humoral immunologic abnormalities (5). 
At present, next generation sequencing gives the opportunity to look for less common variants with large effects. Prompted by our recent genetic analysis of a well-characterized, three-generation Greek family with seven affected women with surgically confirmed endometriosis (6), we performed a whole exome sequencing (WES) in an attempt to identify rare, diseaseassociated exonic variants. In the present study, we report a unique case of a woman of this family with endometriosis, manifesting a co-morbidity of 13 non-gynecological diseases, including SLE, ankylosing spondylitis (AS), MS, bronchial asthma, Crohn's disease (CD), urinary bladder diverticulum, osteoporosis, multinodular goiter, cardiovascular disease, gastroesophageal reflux disease, malignant tumor of urinary bladder, Barrett's esophagus and bilateral cataract, whose history included infertile members suffering from endometriosis as well (Table I). We also explored the potential explanations for the genetic associations between a high number of genes and various diseases, based on the data detected by WES.

\section{Materials and methods}

Participants. We describe the case of a familial case of a 67-year-old female, who presented with a history of endometriosis and underwent surgical hysterectomy at 32 years of age, due to stage IV endometriosis (6). Of interest, her mother had given birth to four children who had no gynecologic problems (6). According to her obstetric and medical history she gave birth to three female offspring and, furthermore, she underwent four surgeries for endometriosis (Table I). All three daughters were diagnosed surgically (laparotomy or laparoscopy) with endometriosis, and the disease was confirmed histologically from biopsies. Moreover, her grandaughters acquired endometriosis.

The age, gynecological and major non-gynecological chronic diseases are shown in Table I. Osteoporosis, recurrent cystitis and urinary bladder diverticulum were evident after total hysterectomy with bilateral salpingo oophorectomy. At the age of 45, she acquired multinodular goiter. Two years later, the patient experienced the first episode of cardiovascular disease. At the age of 50, SLE was diagnosed, while within the next 12 years, four more autoimmune disorders were confirmed, namely AS, MS, bronchial asthma and CD. Apart from the aforementioned autoimmune diseases, the woman experienced malignant tumor of urinary bladder during the sixth decade of her life. The pathology report demonstrated transitional cell carcinoma and the patient underwent intrabladder chemotherapy cycles. The data were collected by the clinicians and pathologists reported the medical records, including surgical procedures and findings. The Ethics Committees of the Human Research at Venizeleio General Hospital of Heraklion (ECHR no.46/6686) (Heraklion, Greece) approved the overall study and a written informed consent was obtained from the patient.

Whole exome sequencing. Genomic DNA was isolated from peripheral blood leukocytes by using the commercial kit (PureLink ${ }^{\circledR}$ Genomic DNA mini kit; Invitrogen Life Technologies; Thermo Fisher Scientific, Inc., Waltham, MA, USA) according to the manufacturer's protocol. Exome sequencing was conducted using the AmpliSeq technology on Ion Proton platform (Thermo Fisher Scientific, Inc.). After sequence assembly using Torrent software, variant annotation was performed using ANNOVAR (hg19 reference; http:// annovar.openbioinformatics.org/en/latest/). Variants were determined using Ion Proton protocol and confirmed using the Genome Analysis Toolkit pipeline.

\section{Results}

WES detected autoimmune disease-risk genotypes. The woman under study was found to be homozygous for the risk allele ' $\mathrm{T}$ ' of the functional, SLE-, AS- and CD-associated rs2476601 SNP of PTPN22 gene, coding for protein tyrosine phosphatase, non-receptor type 22 (Table II). The protein coded by PTPN22 gene contributes to the modulation of negative T-cell selection in the thymus and downregulation of autoreactive T cells in the periphery (7).

The patient under study was heterozygous for both rs 27434 and rs30187 SNPs of ERAP1 gene, T/C and A/G, respectively. $E R A P 1$ gene encodes an endoplasmic reticulum aminopeptidase involved in peptide trimming before HLA class I presentation and has been associated with AS by GWAS (8) as well as CD (9). She was also heterozygous (A/G) for rs602662 FUT2 SNP. FUT2 codes for a protein that is responsible for secretion of the $\mathrm{ABO}$ histo-blood group antigens in the mucosa. Importantly, the heterozygous genotype $A / G$ of rs602662 SNP has been previously associated with SLE, CD and AS $(9,10)$ with all three diseases appearing in the woman under investigation.

Furthermore, WES results showed that this woman was heterozygous for rs10781499 and rs4077515 SNPs of CARD9 as well as rs3197999 SNP of MST1 gene, which have been associated with CD (9-11). Caspase recruitment domain (CARD) 9 is an important adapter protein that is widely expressed in various tissues including liver, spleen, bone marrow, brain, lung and peripheral blood and is closely associated with immune and inflammatory responses (12). MST1 is the basic gene for regulating cell apoptosis in Mammalian ste20-like protein kinase (MST) protein family (13).

Interleukin 16 (IL16), which is also known as a lymphocyte chemoattractant factor, is a proinflammatory cytokine playing a decisive role in various immune and inflammatory responses, while it has also been referred as a causative factor for endometriosis and SLE (14), probably by resulting in the aberrant expression of IL16. The woman under study was found to be heterozygous (G/T) for this SNP and, interestingly, G/T genotype of this SNP has been associated previously with SLE as well as CAD (15), thus indicating that this polymorphism may contribute to the development of both diseases in this case.

WES data for non-autoimmune diseases. Concerning the malignant tumor of urinary bladder identified in the patient, she was found to be heterozygous for rs10936599 SNP of MYNN gene located in 3q26.2 (Table II), an SNP that has been confirmed by GWAS to be a disease-associated gene and may be used for the measure of inherited risk for bladder cancer (BC) (16).

The woman under study was diagnosed with recurrent cystitis at the age of 33 years. Moreover, bronchial asthma manifested at the age of 62 years and was found to be homozygous A/A for the risk allele of rs6967330 of CDHR3 gene, 
Table I. Summary of gynecological and non-gynecological diseases in a patient with endometriosis.

\begin{tabular}{|c|c|}
\hline Age (years) & Obstetric and medical history \\
\hline \multirow[t]{8}{*}{67} & Date of birth: 1951 \\
\hline & Menarche at the age of 11 years, regular menstrual cycles $26-28$ days \\
\hline & At the age of 15 years, first daughter (vaginal delivery) \\
\hline & At the age of 19 years, second daughter (vaginal delivery) \\
\hline & At the age of 25 years, third daughter (vaginal delivery) \\
\hline & +2 miscarriages $<10$ weeks \\
\hline & No history of alcohol and smoking \\
\hline & Co-morbidities with non-gynecological chronic disorders \\
\hline \multirow[t]{2}{*}{27} & Diagnosis of endometriosis, bilateral endometrioma \\
\hline & Between 27 and 32 years she underwent 4 surgeries for endometriosis \\
\hline 32 & Total hysterectomy with bilateral salpingo oophorectomy \\
\hline 33 & Recurrent cystitis, diagnosis and operation of urinary bladder diverticulum \\
\hline 38 & Osteoporosis \\
\hline 45 & Multinodular goiter \\
\hline 47 & Cardiovascular disease \\
\hline 50 & Systemic lupus erythematosus \\
\hline 52 & Ankylosing spondylitis \\
\hline 54 & Multiple sclerosis \\
\hline 57 & Gastro-esophageal reflux disease, diaphragmatocele \\
\hline \multirow[t]{2}{*}{60} & Unilateral macular hole surgery in the eye \\
\hline & Within 3 years, diagnosis and operation of bilateral cataract \\
\hline \multirow[t]{2}{*}{62} & Crohn's disease, confirmed with biopsy \\
\hline & Bronchial asthma \\
\hline 66 & Malignant tumor of urinary bladder: Transitional cell carcinoma \\
\hline 67 & Barrett's esophagus \\
\hline
\end{tabular}

encoding cadherin-related family member 3 which is highly expressed in airway epithelium, which has been associated with an increased susceptibility for asthma (17).

Apparently, numerous genotypes carrying the minor allele of already known SNPs as well as new gene polymorphisms (SNPs and indels) were detected through the conducted WES analysis. However, these data are not presented in detail considering that any speculation regarding a causative association of the observed polymorphisms with the disease pathogenesis has to be confirmed first by functional experiments.

\section{Discussion}

Over a period of 30 years, 1,000 patients with endometriosis from two different geographic locations (USA and Greece) underwent surgical treatment for endometriosis by our colleagues. To the best of our knowledge and upon reviewing the literature, the woman under investigation in the present study represents the first reported case of familial endometriosis associated with 13 non-gynecological diseases. Thus, the woman acquired various non-gynecological conditions including cardiovascular disease, autoimmune diseases and non-gynecological cancer. Our overall findings suggest that women with severe familial endometriosis seem to have a higher risk of developing other chronic disorders.
Interestingly, there are several epidemiological reports regarding the association of endometriosis with serious chronic diseases. We previously confirmed a correlation between endometriosis and asthma, in a group of 55 adolescent girls (18), as well as with various autoimmune diseases, such as rheumatoid arthritis, hypothyroidism, SLE and MS (19). Thus far, laparoscopically-confirmed endometriosis has been significantly associated with subsequent SLE but it remains unclear whether endometriosis itself, hysterectomy or any unidentified factors are related to the increased risk of SLE.

Notably, no history of smoking or alcohol consumption was reported for this woman. Considering that alcohol and smoking affect the development of many diseases evident in this patient i.e., bronchial asthma, BC, cardiovascular disease, it can be assumed that the genetic background rather than environmental life-style factors is of higher contribution in this case, thus adding more weight to the genetic contribution. It is worth noting given that there is a positive correlation between endometriosis and interstitial cystitis (IC) and between IC and BC. A hypothesis that may explain much of the patient's profile, suggests that endometriosis, IC, CD and airway problems (bronchial asthma) are linked to loss of epithelial/mesothelial barrier integrity (20) and, therefore, inflammation could be considered as a natural immunologic response to this sensitivity. 
Table II. Disease-associated genotypes for co-morbidities in the patient with endometriosis

\begin{tabular}{|c|c|c|c|c|c|}
\hline Disease & dbSNP ID & Genes & Function & Genotype & Refs. \\
\hline SLE, AS, CD & rs2476601 & PTPN22 & $\begin{array}{l}\text { T-cell signaling; } \\
\text { macrophage polarization; } \\
\text { interferon signaling }\end{array}$ & TT & (7) \\
\hline $\mathrm{AS}, \mathrm{CD}$ & rs27434 & ERAPI & Antigen presentation & $\mathrm{CT}$ & (9) \\
\hline $\mathrm{AS}, \mathrm{CD}$ & rs30187 & ERAP1 & Antigen presentation & $\mathrm{AG}$ & (10) \\
\hline SLE, AS, CD & rs602662 & FUT2 & $\begin{array}{l}\mathrm{A} \text { and } \mathrm{B} \text { antigen synthesis } \\
\text { pathway }\end{array}$ & $\mathrm{AG}$ & $(10,11)$ \\
\hline SLE, CAD & rs 11556218 & IL16 & $\begin{array}{l}\text { Chemoattractant; } \\
\text { modulator of T-cell } \\
\text { activation }\end{array}$ & GT & $(12,13)$ \\
\hline $\mathrm{CD}$ & rs 10781499 & CARD 9 & $\begin{array}{l}\text { Activation of pro- and } \\
\text { anti-inflammatory } \\
\text { cytokines }\end{array}$ & CT & $(10,11)$ \\
\hline $\mathrm{CD}$ & rs 4077515 & CARD9 & $\begin{array}{l}\text { Activation of pro- and } \\
\text { anti-inflammatory } \\
\text { cytokines }\end{array}$ & $\mathrm{AG}$ & $(10,11)$ \\
\hline $\mathrm{CD}$ & rs3197999 & MST1 & $\begin{array}{l}\text { Regulation of cell } \\
\text { apoptosis }\end{array}$ & CT & (11) \\
\hline $\mathrm{BC}$ & rs 10936599 & $M Y N N$ & $\begin{array}{l}\text { Control of gene } \\
\text { expression }\end{array}$ & CT & $(12,14)$ \\
\hline Bronchial asthma & rs6967330 & CDHR3 & Calcium ion binding & AA & (13) \\
\hline
\end{tabular}

SLE, systemic lupus erythematosus; AS, ankylosing spondylitis; CD, Crohn's disease; CAD, cardiovascular disease; BC, bladder cancer.

Autoimmune diseases are complex diseases characterized by loss of self-tolerance, causing immune-mediated tissue destruction. A similar immunological alteration occurs in endometriosis, thus increasing the number and cytotoxicity of macrophages and leading to various abnormalities in the function and concentrations of $\mathrm{B}$ and $\mathrm{T}$ lymphocytes (21). Endometriosis has been widely characterized as an inflammatory disease, showing a significant deregulation of the immune system at the injury site as well as the uterine cavity of women with endometriosis. Thus, there is growing evidence that women who experience one autoimmune disease are more likely to have additional autoimmune problems, probably due to underlying shared pathogenic pathways. Although the accumulating evidence that common genetic factors might predispose to multiple autoimmune diseases, there are no direct evidences to support clear causality implicating common genetic or environmental factors. Autoimmune diseases developed in the woman under discussion affect predominantly middle-aged women (35-45 years).

As far as the ocular complications are concerned, idiopathic macular hole usually appears after the age of 65 years, with $2 / 3$ of the patients being women and is usually unilateral. Regarding cataract formation, it is known to mostly be related to older age, though other causes exist such as ocular trauma and ocular surgery, radiation, diabetes and prolonged steroid use as well as environmental and genetic predisposition (22).

In conclusion, considering that the advent of highthroughput sequencing technologies has markedly expanded knowledge of genome-wide gene abnormalities, the WES data analysis supports the notion that endometriosis and various autoimmune diseases share possible common genetic and pathophysiological features. The notable number of co-morbidities observed reinforces the necessity for further studies, given that the high number of co-morbidities raises the question of a shared molecular/genetic pathogenic mechanism. If future studies confirm relationships between endometriosis and chronic diseases and/or identify more risk factors predisposing endometriosis patients to develop many non-gynecological diseases, the new findings will have important implications in the management and care of women with endometriosis by allowing physicians to make decision regarding relevant therapeutic approaches.

\section{Acknowledgements}

We would like to thank all the practitioners for providing the data and pathology reports used in this study.

\section{Funding}

Funding Juneau Biosciences provided funding for the sequencing.

\section{Availability of data and materials}

All data relating to the study are available within the manuscript. 


\section{Authors' contributions}

CM, MM, IM, DAS and GNG designed the study and drafted the manuscript. AT, RC, GM, GNG and MIZ searched the literature. GM, AT, IM, CM and MM obtained the clinical data. HMA, RC, KW and MIZ performed experiments. CM, RC, MIZ, DAS, GNG and IM analyzed and interpreted the data. GM, KW, HMA, MM, AT, MIZ, IM and critically revised the manuscript.

\section{Ethics approval and consent to participate}

The Ethics Committees of the Human Research at Venizeleio General Hospital of Heraklion (ECHR no.46/6686) (Heraklion, Greece) approved the overall study and written informed consent was obtained from the patient.

\section{Patient consent for publication}

Not applicable.

\section{Competing interests}

HMA, RC and KW are employed by Juneau Biosciences who provided salary support for the study. Demetrios A. Spandidos is the Editor-in-Chief for the journal, but had no personal involvement in the reviewing process, or any influence in terms of adjudicating on the final decision, for this article.

\section{References}

1. Halis G and Arici A: Endometriosis and inflammation in infertility. Ann N Y Acad Sci 1034: 300-315, 2004.

2. Sapkota Y, Steinthorsdottir V, Morris AP, Fassbender A, Rahmioglu N, De Vivo I, Buring JE, Zhang F, Edwards TL, Jones S, et al; iPSYCH-SSI-Broad Group: Meta-analysis identifies five novel loci associated with endometriosis highlighting key genes involved in hormone metabolism. Nat Commun 8: 15539, 2017. 10.1038/ncomms15539.

3. Choi EJ, Cho SB, Lee SR, Lim YM, Jeong K, Moon H-S and Chung H: Comorbidity of gynecological and non-gynecological diseases with adenomyosis and endometriosis. Obstet Gynecol Sci 60: 579-586, 2017.

4. Alviggi C, Carrieri PB, Pivonello R, Scarano V, Pezzella M, De Placido G, Colao A and Matarese G: Association of pelvic endometriosis with alopecia universalis, autoimmune thyroiditis and multiple sclerosis. J Endocrinol Invest 29: 182-189, 2006.

5. Pasoto SG, Abrao MS, Viana VST, Bueno C, Leon EP and Bonfa E: Endometriosis and systemic lupus erythematosus: A comparative evaluation of clinical manifestations and serological autoimmune phenomena. Am J Reprod Immunol 53: 85-93, 2005

6. Matalliotakis M, Zervou MI, Matalliotaki C, Arici A, Spandidos DA, Matalliotakis I and Goulielmos GN: Genetic association study in a three-generation family with seven members with endometriosis. Mol Med Rep 16: 6077-6080, 2017.

7. Criswell LA, Pfeiffer KA, Lum RF, Gonzales B, Novitzke J, Kern M, Moser KL, Begovich AB, Carlton VE, Li W, et al: Analysis of families in the multiple autoimmune disease genetics consortium (MADGC) collection: The PTPN22 620W allele associates with multiple autoimmune phenotypes. Am J Hum Genet 76: 561-571, 2005 .
8. Australo-Anglo-American Spondyloarthritis Consortium (TASC); Reveille JD, Sims AM, Danoy P, Evans DM, Leo P, Pointon JJ, Jin R, Zhou X and Bradbury LA: Genome-wide association study of ankylosing spondylitis identifies non-MHC susceptibility loci. Nat Genet: Jan 10, 2010 (Epub ahead of print). doi: 10.1038/ng.513.

9. Franke A, McGovern DP, Barrett JC, Wang K, Radford-Smith GL, Ahmad T, Lees CW, Balschun T, Lee J, Roberts R, et al: Genome-wide meta-analysis increases to 71 the number of confirmed Crohn's disease susceptibility loci. Nat Genet 42: 1118-1125, 2010.

10. Tong M, McHardy I, Ruegger P, Goudarzi M, Kashyap PC, Haritunians T,Li X, Graeber TG, Schwager E, Huttenhower C, et al: Reprograming of gut microbiome energy metabolism by the FUT2 Crohn's disease risk polymorphism. ISME J 8: 2193-2206, 2014.

11. Palmieri O, Bossa F, Valvano MR, Corritore G, Latiano T, Martino G, D'Incà R, Cucchiara S, Pastore M, D'Altilia M, et al: Crohn's disease localization displays different predisposing genetic variants. PLoS One 12: e0168821, 2017.

12. Gross O, Gewies A, Finger K, Schäfer M, Sparwasser T, Peschel C, Förster I and Ruland J: Card9 controls a non-TLR signalling pathway for innate anti-fungal immunity. Nature 442: Jul 12, 2006 (Epub ahead of print). doi: 10.1038/nature04926.

13. Ma Y, Yang Y, Wang F, Wei Q and Qin H: Hippo-YAP signaling pathway: A new paradigm for cancer therapy. Int J Cancer 137: Jul 22, 2014 (Epub ahead of print). doi: 10.1002/ijc.29073.

14. Xue H, Gao L, Wu Y, Fang W, Wang L, Li C, Li Y, Liang W and Zhang L: The IL-16 gene polymorphisms and the risk of the systemic lupus erythematosus. Clin Chim Acta 403: Mar 17, 2009 (Epub ahead of print). doi: 10.1016/j.cca.2009.03.016.

15. Wu J, Wang Y, Zhang Y and Li L: Association between interleukin-16 polymorphisms and risk of coronary artery disease. DNA Cell Biol 30: Jan 8, 2011 (Epub ahead of print). doi: 10.1089/ dna.2010.1145

16. de Maturana EL, Rava M, Anumudu C, Sáez O, Alonso D and Malats N: Bladder cancer genetic susceptibility. A systematic review. Bladder Cancer 4: 215-226, 2018.

17. Bønnelykke K, Sleiman P, Nielsen K, Kreiner-Møller E, Mercader JM, Belgrave D, den Dekker HT, Husby A, Sevelsted A, Faura-Tellez G, et al: A genome-wide association study identifies CDHR3 as a susceptibility locus for early childhood asthma with severe exacerbations. Nat Genet 46: Nov 17, 2013 (Epub ahead of print). doi: $10.1038 /$ ng.2830.

18. Matalliotakis M, Goulielmos GN, Matalliotaki C, Trivli A, Matalliotakis I and Arici A: Endometriosis in adolescent and young girls: Report on a series of 55 cases. J Pediatr Adolesc Gynecol 30: May 27, 2017 (Epub ahead of print). doi: 10.1016/j. jpag.2017.05.007.

19. Matalliotakis IM, Cakmak H, Fragouli YG, Goumenou AG, Mahutte NG and Arici A: Epidemiological characteristics in women with and without endometriosis in the Yale series. Arch Gynecol Obstet 277: Oct 9, 2007 (Epub ahead of print). doi: 10.1007/s00404-007-0479-1.

20. Albertsen HM and Ward K: Genes linked to endometriosis by GWAS are integral to cytoskeleton regulation and suggests that mesothelial barrier homeostasis is a factor in the pathogenesis of endometriosis. Reprod Sci 24: Jul 28, 2016 (Epub ahead of print). doi: $10.1177 / 1933719116660847$.

21. Matarese G, De Placido G, Nikas Y and Alviggi C: Pathogenesis of endometriosis: Natural immunity dysfunction or autoimmune disease? Trends Mol Med 9: 223-228, 2003.

22. Prokofyeva E, Wegener A and Zrenner E: Cataract prevalence and prevention in Europe: A literature review. Acta Ophthalmol 91: Jun 20, 2012 (Epub ahead of print). doi: 10.1111/j.1755-3768. 2012.02444.x. 